\title{
Erratum to: A novel thermophilic endo- $\beta$-1,4-mannanase from Aspergillus nidulans XZ3: functional roles of carbohydrate-binding module and Thr/Ser-rich linker region
}

\author{
Haiqiang Lu • Huiying Luo • Pengjun Shi • Huoqing Huang • \\ Kun Meng • Peilong Yang $\cdot$ Bin Yao
}

Published online: 18 December 2014

(C) Springer-Verlag Berlin Heidelberg 2014

Erratum to: Appl Microbiol Biotechnol (2014)

DOI 10.1007/s00253-013-5112-6

The original version of this article contains an error.

The authors would like to amend the text on page 2158 (paragraph 3) such that instead of:

Nucleotide sequence accession numbers

The nucleotide sequence for the GH5 endo- $\beta$-1,4-mannanase gene (man $5 X Z 3)$ of $A$. nidulans XZ3 were deposited into the GenBank database under the accession number JX297422 (http://www.ncbi.nlm.nih.gov/nuccore/459586151).

The nucleotide sequence for the ITS gene of $A$. nidulans XZ3 was $100 \%$ identity with $18 \mathrm{~S}$ rRNA gene of Aspergillus nidulans, isolate YESM 10 (the accession number LM653124).

The online version of the original article can be found at:http://dx.doi.org/ 10.1007/s00253-013-5112-6.

H. Lu $\cdot$ H. Luo $\cdot$ P. Shi $\cdot$ H. Huang $\cdot$ K. Meng $\cdot$ P. Yang $\cdot$ B. Yao $(\bowtie)$

Key Laboratory for Feed Biotechnology of the Ministry of Agriculture, Feed Research Institute, Chinese Academy of Agricultural Sciences, No. 12 Zhongguancun South Street, Beijing 100081, People's Republic of China

e-mail: binyao@caas.cn 